\title{
Repulsive Casimir force in Bose-Einstein Condensate
}

\author{
Mir Mehedi Faruk ${ }^{1 *}$ and Shovon Biswas ${ }^{2}$ \\ McGill University Montreal, QC H3A 2T8, Canada ${ }^{1}$ \\ Department of Electrical and Electronic Engineering, Bangladesh University of Engineering and \\ Technology, Dhaka 1000, Bangladesh. ${ }^{2}$
}

December 25, 2017

\begin{abstract}
We study the Casimir effect for a three dimensional system of ideal free massive Bose gas in a slab geometry with Zaremba and anti-periodic boundary conditions. It is found that for these type of boundary conditions the resulting Casimir force is repulsive in nature, in contrast with usual periodic, Dirichlet or Neumann boundary condition where the Casimir force is attractive (Martin P. A. and Zagrebnov V. A., Europhys. Lett., 73 (2006) 15.). Casimir forces in these boundary conditions also maintain a power law decay function below condensation temperature and exponential decay function above the condensation temperature albeit with a positive sign, identifying the repulsive nature of the force.
\end{abstract}

\section{Introduction}

In his original paper, Casimir described a nonclassical attractive force related to quantum vacuum fluctuations in the electromagnetic field between two uncharged parallel conducting plates 1. Since then, the Casimir effect for quantum vacuum fluctuation has been extensively studied for various types of geometries and boundary conditions (see ref. 2, 3] and the references therein) using Quantum Field Theory (QFT) techniques. But the Casimir type force due to thermal fluctuation in ideal free Bose gas in vacuum was first reported in the seminal work of Martin and Zagrebnov 4 and since then it has been thoroughly studied [5, 6, 7, 8, 9, 10, in Statistical Mechanics (SM). It is quite well known that the Casimir force depends upon boundary conditions and is attractive for scalar fields (as well as free Bose gas) in either case of vacuum or thermal fluctuation and is reported to be an attractive force for the usual case of Dirichlet $(\mathrm{D})$, Neumann $(\mathrm{N})$ as well as periodic boundary $(\mathrm{P})$ conditions on both sides. However, it is of significant interest to get physical configurations where the Casimir force is repulsive instead of attractive, not only for its relevance for technical applications to nano devices [3, 11, 12, 13, 14, but also because the existence of repulsive or null Casimir forces allows a more accurate analysis of micro-gravity effects [15] as well as the study of cosmic strings [16. It has been recently reported that in QFT approach one can achieve repulsive Casimir force due to quantum fluctuation using Zaremba1 1 and anti-periodic boundary condition [17, 18, (see section 3.4 and 3.5 of ref. [18). But Casimir type force due to thermal fluctuation in free Bose gas with these type of boundary condition is yet to be reported. Casimir-type interactions are nowadays identified in several types of systems spanning from biology 19 to cosmology 20 but the QED and condensed-matter contexts are those where the theoretical predictions concerning the existence and properties of Casimir forces found firm experimental confirmation 21, 22. Therefore, it is of significant importance to figure out if Zaremba and anti-periodic boundary conditions can be responsible for repulsive Casimir force in a free Bose gas due to thermal fluctuations in SM. In this manuscript we have considered an ideal free Bose gas confined in three dimensional slab like geometry $L \times L \times d$ (where $L>>d$ ), subjected to Zaremba/anti-periodic boundary condition in $z$ direction and calculated the repulsive Casimir force due to thermal fluctuation in vacuum. Point to note, imperfect Bose gas with the repulsive microscopic interparticle interactions subjected to periodic boundary conditions generate an effective Casimir force of repulsive nature [10. But in that case [10, the repulsive behaviour of Casimir force is due to the interparticle interaction whereas in the present endeavor the repulsive Casimir force is solely due to boundary condition. Relation between the decay length characterizing the Casimir force (due to these boundary condition) and

\footnotetext{
*Corresponding author: muturza3.1416@gmail.com, mir.faruk@mail.mcgill.ca

${ }^{1}$ Zaremba boundary condition indicates Dirichlet condition in one side and Neumann condition in another side, also known as hybrid or mixed boundary condition.
} 
the bulk correlation lengths are also discussed.

\section{Model}

Let us consider ideal free massive Bose gas confined between two infinitely large square shaped plates of area $A$. The plates are along the $x y$ plane and are separated by distance $d$ along the z-axis. For the slab geometry we consider $\sqrt{A}>>d$ as well the system is in thermodynamic equilibrium with its surroundings at temperature $T$. At this temperature the thermal de Broglie wavelength of a single particle of mass $m$ is $\lambda=\hbar \sqrt{\beta / m}$ where $\beta=\frac{1}{k_{B} T}$ and $k_{B}$ is the Boltzmann's constant. In the thermodynamic limit, we consider $\lambda<<d$. Thus the energy of the single particle is $E=\frac{q_{x}^{2}}{2 m}+\frac{q_{y}^{2}}{2 m}+\frac{p_{z}^{2}}{2 m}$, where for Zaremba (Z) and antiperiodic (A) boundary condition [17, 18] we have respectively,

$$
\begin{aligned}
& p_{z}=\left(n+\frac{1}{2}\right) \frac{\hbar \pi}{d}, \quad n=0,1,2,3, \ldots \\
& p_{z}=\left(n+\frac{1}{2}\right) \frac{2 \pi \hbar}{d}, \quad n=0, \pm 1, \pm 2, \pm 3, \ldots
\end{aligned}
$$

Based on the assumptions described above the grand-canonical potential per unit area can be written as

$$
\Phi_{d}(T, \mu)=\beta^{-1} \sum_{n=0}^{\infty} \int_{-\infty}^{\infty} \int_{-\infty}^{\infty} \frac{d q_{x} d q_{y}}{(2 \pi \hbar)^{2}} \Omega\left(q_{x}, q_{y}, p_{z}\right)
$$

where,

$$
\Omega=\left\{\begin{array}{l}
\ln \left[1-e^{-\beta\left(\frac{q_{x}^{2}}{2 m}+\frac{q_{y}^{2}}{2 m}+\frac{\pi^{2} \hbar^{2}\left(n+\frac{1}{2}\right)^{2}}{2 m d^{2}}-\mu\right)}\right] \\
\ln \left[1-e^{-\beta\left(\frac{q_{x}^{2}}{2 m}+\frac{q_{y}^{2}}{2 m}+\frac{2 \pi^{2} \hbar^{2}\left(n+\frac{1}{2}\right)^{2}}{m d^{2}}-\mu\right)}\right]
\end{array}\right.
$$

Here $\mu$ is the chemical potential. Representing (2) by its low-activity series for $\mu<0$ and performing the integration we obtain,

$$
\Phi_{d}(T, \mu)=\left\{\begin{array}{l}
-\frac{1}{2 \pi \beta \lambda^{2}} \sum_{r=1}^{\infty} \frac{e^{\beta r \mu}}{r^{2}} \sum_{n=0}^{\infty} e^{-\pi\left(n+\frac{1}{2}\right)^{2}\left(r \pi(\lambda / d)^{2}\right) / 2} \\
-\frac{1}{2 \pi \beta \lambda^{2}} \sum_{r=1}^{\infty} \frac{e^{\beta r \mu}}{r^{2}} \sum_{n=0}^{\infty} e^{-\pi\left(n+\frac{1}{2}\right)^{2}\left(r \pi(2 \lambda / d)^{2}\right) / 2}
\end{array}\right.
$$

The following identities can be established from Poisson summation formula [4] (see appendix),

$$
\sum_{n=0}^{\infty} e^{-\pi\left(n+\frac{1}{2}\right)^{2} a}=\frac{1}{2 \sqrt{a}}+\sum_{n=1}^{\infty}(-1)^{n} e^{-\pi n^{2} / a}, \quad a>0
$$

and

$$
\sum_{n=-\infty}^{\infty} e^{-\pi\left(n+\frac{1}{2}\right)^{2} a}=\frac{1}{\sqrt{a}}+2 \sum_{n=1}^{\infty}(-1)^{n} e^{-\pi n^{2} / a}, \quad a>0
$$

So, $\Phi_{d}(T, \mu)$ can therefore be expressed as follows,

$$
\Phi_{d}(T, \mu)=\Phi_{b u l k}(T, \mu)+\Phi_{C a s}(T, \mu)
$$

In both cases the bulk contribution to grand potential is,

$$
\Phi_{b u l k}=-\frac{d}{\beta(\sqrt{2 \pi} \lambda)^{3}} \sum_{r=1}^{\infty} \frac{e^{\beta r \mu}}{r^{5 / 2}}
$$


whereas, the second term is different for cases,

$$
\Phi_{C a s}(T, \mu)=-q \times\left\{\begin{array}{l}
\sum_{n=1}^{\infty} \sum_{r=1}^{\infty} \frac{e^{\beta r \mu}}{r^{5 / 2}}(-1)^{n} e^{-2(n d / \lambda)^{2} / r} \\
\sum_{n=1}^{\infty} \sum_{r=1}^{\infty} \frac{e^{\beta r \mu}}{r^{5 / 2}}(-1)^{n} e^{-2(n d / 2 \lambda)^{2} / r}
\end{array}\right.
$$

where $q=\frac{2 d}{\beta(\sqrt{2 \pi} \lambda)^{3}}$. Note that, the surface term is absent for both cases just like periodic boundary condition 4 . Nevertheless, in any general system the bulk as well the surface term, do not contribute to the Casimir force, because the force due to the bulk term is counterbalanced by the same contribution acting from outside the slabs when they are immersed in the critical medium [4, 6] and the surface term does not change with the change of thickness of the slab. Now the Casimir force can be obtained from Casimir potential through $-\partial_{d} \Phi_{C a s}^{(Z)}$. Now let us consider two separate cases: condensed and noncondensed case subject to Zaremba boundary condition-

In condensed phase $T \leq T_{c}(\mu=0)$, BEC occurs in Bose gas and $\mu=0$. We write eq. (9) as

$$
\Phi_{\text {Cas }}^{(Z)}(T, 0)=-\frac{2}{\beta(\sqrt{2 \pi})^{3} d^{2}} \sum_{n=1}^{\infty}(-1)^{n}(\lambda / d)^{2} \sum_{r=1}^{\infty} \psi_{n}\left((\lambda / d)^{2} r\right)
$$

where we have defined $\psi_{n}(x)=\frac{e^{-2 n^{2} / x}}{x^{5 / 2}}$. We now use the fact. $\lambda / d<<1$ and the sum $\sum_{r=1}^{\infty}$ can be converted into an integral. Therefore we obtain,

$$
\Phi_{\text {Cas }}^{(Z)}(T, 0)=-\frac{1}{8 \pi \beta d^{2}} \sum_{n=1}^{\infty} \frac{(-1)^{n}}{n^{3}}=\frac{\eta(3)}{8 \pi \beta d^{2}}
$$

Where $\eta(s)=\sum_{n=0}^{\infty} \frac{(-1)^{n-1}}{n^{s}}$ is the Dirichlet eta function. Now, using the relation, $\eta(3)=\frac{3}{4} \zeta(3)$, where $\zeta(s)=\sum_{n=1}^{\infty} n^{-s}$ is the Riemann zeta function. We finally obtain

$$
\Phi_{\text {Cas }}^{(Z)}(T, 0)=\frac{3 \zeta(3)}{32 \pi} \frac{k_{B} T}{d^{2}}
$$

Note that the sign of $\Phi_{\text {Cas }}^{(Z)}(T, 0)$ is positive in contrast to the situation described in references [4. Finally we have the Casimir force per unit area:

$$
F_{c}=-\partial_{d} \Phi_{C a s}^{(Z)}=\frac{3 \zeta(3)}{16 \pi} \frac{k_{B} T}{d^{3}} .
$$

which is repulsive.

Finally we consider the non-condensed phase with $T>T_{c}(\mu<0)$. The double sums in eq. (6) for $d>>\lambda$ can be estimated following Ref 4 ]

$$
\sum_{n=1}^{\infty} \sum_{r=1}^{\infty} \frac{e^{\beta r \mu}}{r^{5 / 2}}(-1)^{n} e^{-2(n d / \lambda)^{2} / r} \leq \frac{\zeta(5 / 2)}{e^{\sqrt{-8 \beta \mu} d / \lambda}+1}=-O\left(e^{-\sqrt{-8 \beta \mu} d / \lambda}\right) .
$$

As a result, the leading contributing to Casimir potential (eq 6) is decaying exponentially just like Periodic, Dirichlet or Neumann boundary condition 4 , but the force is positive unlike those boundary condition. The Casimir force in noncondensed phase is therefore $F_{C} \propto \exp \left(-d / \kappa^{(Z)}\right)$, where

$$
\kappa^{(Z)}=\frac{\lambda}{4} \sqrt{\frac{2}{(-\mu) \beta}}
$$

The Casimir decay length, $\kappa$ for mixed boundary condition is exactly equal to the Dirichlet/Neumann $[8]$ case 2 . Lets turn our attention towards the other case with anti-periodic boundary condition. Following the same procedure we find out the Casimir force for $T<T_{c}$ is,

$$
F_{c}^{(A)}=-\partial_{d} \Phi_{C a s}^{(A)}=\frac{3 \zeta(3)}{2 \pi} \frac{k_{B} T}{d^{3}} .
$$

\footnotetext{
${ }^{2}$ the definition of thermal wavelength with us and reference 8 is different by a factor of $\frac{1}{\sqrt{2 \pi}}$
} 


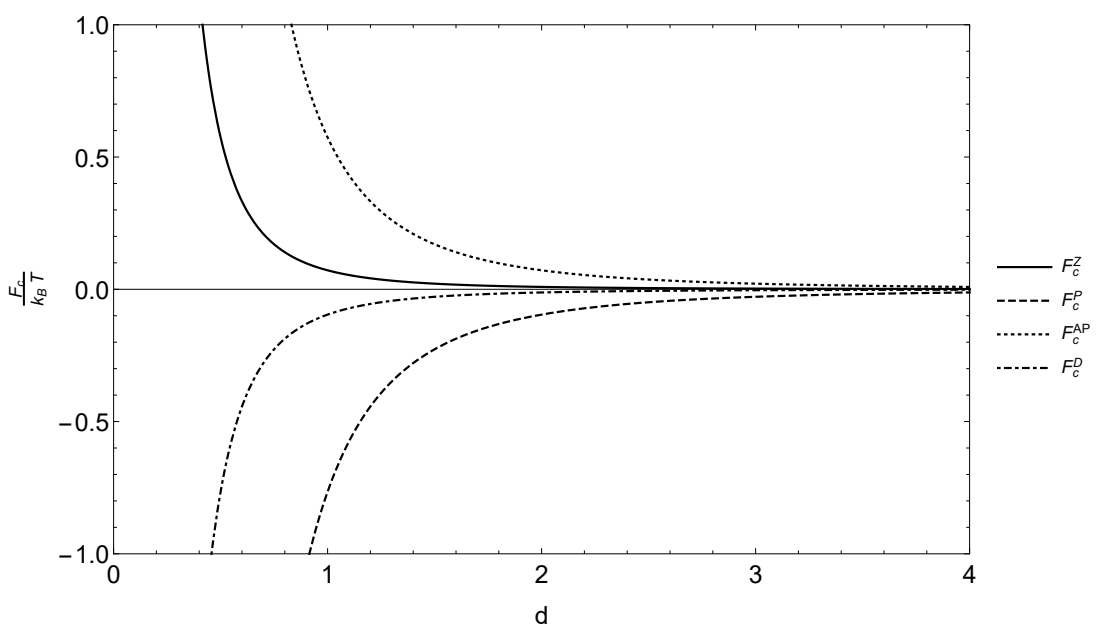

Figure 1: The Casimir force of ideal Bose gas in condensed phase $\left(T<T_{c}\right)$.

And in the non condensed phase the Casimir force is $F_{C}^{(A)} \propto \exp \left(d / \kappa^{(A)}\right)$ with

$$
\kappa^{(A)}=\lambda \sqrt{\frac{1}{2(-\mu) \beta}}
$$

In ref. [8], it has been established that decay length of Casimir force is related directly to the Bulk correlation length for Dirichlet (D), periodic (P) and Neumann (N) boundary conditions, $\frac{1}{2} \kappa^{(P)}=\kappa^{(D)}=\kappa^{(N)}=\xi=\frac{\lambda}{4} \sqrt{\frac{2}{(-\mu) \beta}}$. We have thus extended their relation in case of Zaremba and anti-periodic boundary condition $\frac{1}{2} \kappa^{(P)}=\kappa^{(D)}=\kappa^{(N)}=$ $\kappa^{(Z)}=\frac{1}{2} \kappa^{(A P)}$. But, most significant point of this calculation is the repulsive nature of critical Casimir force, both below and above critical temperature. Nevertheless from here we can see that, like the other boundary conditions upon approaching the phase containing the condensate $(\mu \rightarrow 0)$ the range of force and the correlation length diverges with the critical exponent $\nu=1 / 2$ in Zaremba and anti-periodic boundary condition, identifying the characteristic nature of ideal gas.

\section{Concluding remarks}

In conclusion, we have identified the repulsive nature of Casimir force in both phases of an ideal free Bose gas for thermal fluctuations in vacuum subjected to Zaremba and anti-periodic boundary conditions using the techniques of statistical mechanics. Extracting the final result in the current study is possible due to the identities of eq. (5) and (6) (referred to as Jacobi identity in eq. (16) of ref. [4). If one compares these two identities with the corresponding identities for Neumann, Dirichlet and periodic case, one can immediately notice the $(-1)^{n}$ in the second term with a sum over $n$ in eq (5) and (6). This results in a positive contribution to the Casimir term in grand canonical potential (eq. 12) unlike the cases with Neumann, Dirichlet or periodic boundary condition [4, 5]. The reason behind the $(-1)^{n}$ term in our identities of eq. (5) and (6) is the quantised momenta in eq. (1), which is proportional to the half integers in this case whereas for Dirichlet, Neumann or periodic scenario quantised momenta whch are proportional to the integers. As a consequence, these boundary conditions result in a repulsive Casimir force. In three dimensions, the magnitude of Casimir force with Zaremba boundary condition is $\frac{3}{32}$ times the Casimir force in periodic boundary condition, while for antiperiodic scenario the Casimir force is attenuated by a factor of $\frac{3}{4}$ in comparison to the periodic case for any temperature 3 . But these values will of course change for trapped bosonic systems 6 ] which needs to be investigated as they are substantially related with experimental detection of BEC 26, 27. Point to note, this analysis is done solely for ideal gas without any sort of interaction, which proves that the repulsive behaviour is solely due to boundary condition. But at the same time the effect of interaction needs to be checked under these boundary conditions to find out if any interesting scenario arises like ref [10. This is the next program that we wish to take up. The current study unraveling repulsive Casimir force in Bose Einstein condensate (BEC) could be a good prospect to different aspects of physics including nanotechnology [14, 15], biology [19, and complex networks [28. However, we should investigate the case of dynamical Casimir 2, 3, 23] effect in BEC, which has not yet been reported. Such a

\footnotetext{
${ }^{3}$ the Casimir force for Dirichlet/Neumann boundary condition are $\frac{1}{8}$ times than the periodic one 6
} 
study can not only disentangle new features of BEC but also shed new light on the relationship between Casimir force and the Cosmological constant, Dark energy and Dark matter 24, 25, especially in those models where scalar field BEC 29] and Axion BEC [30] are possible Dark matter candidates.

\section{Appendix}

In this section we derive the mathematical identities described in eq. (5) and (6). For appropriate functions $f$, the Poisson summation formula can be stated as

$$
\sum_{n \in \mathbb{Z}} f(n)=\sum_{n \in \mathbb{Z}} \hat{f}(\nu)
$$

where, $\hat{f}(\nu)$ is the Fourier transform of $f(n)$.

$$
\hat{f}(\nu)=\int_{-\infty}^{\infty} d n f(n) e^{i 2 \pi n \nu}
$$

Then,

$$
\begin{aligned}
\sum_{n=-\infty}^{\infty} e^{-\pi a(n+1 / 2)^{2}} & =\sum_{\nu=-\infty}^{\infty} \int_{-\infty}^{\infty} d n e^{-\pi a(n+1 / 2)^{2}+i 2 \pi n \nu} \\
& =\sum_{\nu=-\infty}^{\infty} \frac{1}{\sqrt{a}} e^{-i \nu \pi-\pi \nu^{2} / a} \\
& =\sum_{n=-\infty}^{\infty} \frac{1}{\sqrt{a}}(-1)^{n} e^{-\pi n^{2} / a} \\
& =\frac{1}{\sqrt{a}}+2 \sum_{n=1}^{\infty} \frac{1}{\sqrt{a}}(-1)^{n} e^{-\pi n^{2} / a}
\end{aligned}
$$

which is equation (6). Now the left hand side of eq. (20) can be re written as,

$$
\begin{aligned}
\sum_{n=-\infty}^{\infty} e^{-\pi a(n+1 / 2)^{2}=} & \underbrace{e^{-\pi a / 4}}_{n=0}+\underbrace{e^{-\pi a(3 / 2)^{2}}}_{n=1}+\underbrace{e^{-\pi a(5 / 2)^{2}}}_{n=2}+\underbrace{e^{-\pi a(7 / 2)^{2}}}_{n=3}+\underbrace{e^{-\pi a(9 / 2)^{2}}}_{n=4}+\ldots \ldots \\
& \underbrace{e^{-\pi a / 4}}_{n=-1}+\underbrace{e^{-\pi a(3 / 2)^{2}}}_{n=-2}+\underbrace{e^{-\pi a(5 / 2)^{2}}}_{n=-3}+\underbrace{e^{-\pi a(7 / 2)^{2}}}_{n=-4}+\underbrace{e^{-\pi a(9 / 2)^{2}}}_{n=-5}+\ldots \ldots
\end{aligned}
$$

Therefore one can see the $n=0$ term matches with $n=-1, n=1$ term matches with $n=-2, n=2$ term matches with $n=-3, n=3$ term matches with $n=4$ and so on. As a result the eq. (21) can be written as,

$$
\begin{aligned}
\sum_{n=-\infty}^{\infty} e^{-\pi a(n+1 / 2)^{2}} & =2(\underbrace{e^{-\pi a / 4}}_{n=0}+\underbrace{e^{-\pi a(3 / 2)^{2}}}_{n=1}+\underbrace{e^{-\pi a(5 / 2)^{2}}}_{n=2}+\underbrace{e^{-\pi a(7 / 2)^{2}}}_{n=3}+\underbrace{e^{-\pi a(9 / 2)^{2}}}_{n=4}+\ldots \ldots) \\
& =2 \sum_{n=0}^{\infty} e^{-\pi\left(n+\frac{1}{2}\right)^{2} a}
\end{aligned}
$$

Combining equation (22) and (20) we obtain,

$$
\sum_{n=0}^{\infty} e^{-\pi\left(n+\frac{1}{2}\right)^{2} a}=\frac{1}{2 \sqrt{a}}+\sum_{n=1}^{\infty}(-1)^{n} e^{-\pi n^{2} / a}
$$

which is eq. (5) 


\section{References}

[1] Casimir H. B. G., Proc. K. Ned. Akad. Wet., 51 (1948) 793.

[2] Milton K. A., The Casimir Effect (World Scientific) 2001

[3] M. Bordag, U.Mohideen, V.M. Mostepanenko, Physics Reports 353 (2001) 1-205.

[4] Martin P. A. and Zagrebnov V. A., Europhys. Lett., 73 (2006) 15.

[5] Biswas S., Eur. Phys. J. D, 42 (2007) 109.; Biswas S., J. Phys. A: Math. Theor., 40 (2007) 9969

[6] Tongling Lin, Guozhen Su, Qiuping A. Wang and Jincan Chen, 2012 EPL 9840010.

[7] Marek Napiórkowski and Jarosław Piasecki Phys. Rev. A 95, 063627.

[8] Marek Napiórkowski, Jarosław Piasecki, J Stat Phys (2014) 156:1136-1145.

[9] Napiórkowski, M., Piasecki, J. Phys. Rev. E 84, 061105 (2011)

[10] P. Jakubczyk, M. Napiorkowski and T. Sek, EPL, 113 (2016) 30006

[11] C. Genet, Lambrecht. Reynaud, Eur. Phys. J. Special Topics 160, 183 (2008);

[12] Kimball A Milton et al 2012 J. Phys. A: Math. Theor. 45374006

[13] Andrea Gambassi 2009 J. Phys.: Conf. Ser. 161012037

[14] O. Kenneth, I. Klich, A. Mann, and M. Revzen, DOI: 10.1103/PhysRevLett.89.033001.

[15] Sandra J. Veen, Oleg Antoniuk, Bart Weber, Marco A. C. Potenza, Stefano Mazzoni, Peter Schall, and Gerard H. Wegdam, Phys. Rev. Lett. 109, 248302. F. M. Serry, D. Walliser, and G. J. Maclay, J. Microelectromech. Syst. 4, 193 (1995); H. B. Chan, V. A. Aksyuk, R. N. Kleiman, D. J. Bishop, and F. Capasso, Science 291, 1941 (2001).

[16] A. A. Saharian, S. Kotanjyan, Eur. Phys. J. C (2011) 71: 1765.

[17] Xiang-hua Zhai and Xin-zhou Li, Phys. Rev. D 76, 047704 (2007).

[18] M. Asorey, J.M. Munoz-Castaneda, Nuclear Physics B 874 (2013) 852-876.

[19] K Bradonjić, J D Swain, A Widom and Y N Srivastava, J. Phys.: Conf. Ser. 161012035.

[20] Gaurang Mahajan, Sudipta Sarkar, T. Padmanabhan, Physics Letters B 641 (2006) 6-10; I. Brevik, K. A. Milton, S. D. Odintsov, and K. E. Osetrin Phys. Rev. D 62, 064005.

[21] Garcia R. and Chan M. H., Phys. Rev. Lett., 83 (1999) 1187. Garcia R. and Chan M. H., Phys. Rev. Lett., 88 (2002) 086101. Fukuto M., Yano Y. F. and Pershan P. S., Phys. Rev. Lett., 94 (2005) 135702.

[22] Ganshin A., Scheidemantel S., Garcia R. and Chan M. H., Phys. Rev. Lett., 97 (2006) 075301. Hartlein C., Helden L., Gambassi A., Dietrich S. and Bechinger C., Nature, 451 (2008) 172.

[23] C. M. Wilson, G. Johansson, A. Pourkabirian, M. Simoen, J. R. Johansson, T. Duty, F. Nori and P. Delsing Nature 479, 376-379.

[24] Kimball A Milton 2004 J. Phys. A: Math. Gen. 37 R209.

[25] Marek Szydłowski and Włodzimierz Godłowski, Int. J. Mod. Phys. D 17, 343 (2008).

[26] C. C. Bradley, C. A. Sackett, J. J. Tollett and R. G. Hulet, Phys. Rev. Lett. 75, 1687, (1995).

[27] M. H. Anderson, J. R. Esher, M. R. Mathews, C. E. Wieman and E. A. Cornell, Science 269, 195, (1995).

[28] Dorogovtsev, S. N.; Mendes, J. F. F. (2001). Phys. Rev. E 63: 056125.

[29] Saurya Das and Rajat K Bhaduri 2015 Class. Quantum Grav. 32105003.

[30] Ozgur Erken, Pierre Sikivie, Heywood Tam, Qiaoli Yang, arXiv:1111.3976. 